\title{
NOTE \\ Postnatal changes in the relative abundance of intestinal Lactobacillus spp. in newborn calves
}

\author{
Tadashi TAKINO ${ }^{1,2)}$, Yuko KATO-MORI ${ }^{1)}$, Daisuke MOTOOKA ${ }^{3)}$, \\ Shota NAKAMURA ${ }^{3)}$, Tetsuya IIDA ${ }^{3)}$ and Katsuro HAGIWARA ${ }^{1)^{*}}$ \\ 1)School of Veterinary Medicine, Rakuno Gakuen University, 582 Bunkyodai, Ebetsu-shi, Hokkaido 069-0836, \\ Japan \\ 2)Scientific Feed Laboratory Co., Ltd., 3-5 Miyahara, Takasaki, Gunma, Japan \\ ${ }^{3)}$ Research Institute for Microbial Diseases, Osaka University, 3-1 Yamadaoka, Suita, Osaka, Japan
}

J. Vet. Med. Sci.

79(3): 452-455, 2017

doi: 10.1292/jvms.16-0406

Received: 25 August 2016

Accepted: 20 December 2016

Published online in J-STAGE:

8 January 2017
ABSTRACT. The intestinal microbiota of newborn calves changes during the early postnatal period and influences their health and immune function. We studied the compositional changes in the intestinal microbiome of newborn calves during the first week after birth by metagenomic analysis. In feces from newborn calves, we identified 4 bacterial phyla, namely, Actinobacteria, Bacteroidetes, Firmicutes and Proteobacteria. The relative abundance of Lactobacillaceae significantly increased from day 1 to day 7 . We evaluated Lactobacillus spp. colony numbers using selective agar plates and confirmed that the abundance of Lactobacillus spp. significantly increased during the first 7 days after birth. In conclusion, Lactobacillus spp. colonized the intestinal tract of calves during the first 7 days after birth.

KEY WORDS: bacterial culture, metagenomics analysis, microbiome

The intestinal microbiota is affected by gender, diet, age, antibiotic treatment and environmental factors. It has been reported that the relative abundance (RA) values of Bifidobacterium and Lactobacillus in the intestinal microbiota vary according to the host species [4]. The period from birth to weaning is a period of great stress to young calves; at this stage, calves undergo the metabolic, nutritional and behavioral changes required to become a functional ruminant $[2,7]$.

Recently, it became possible to perform metagenomic analysis for simultaneous identification of all the taxa comprising microbial communities by calculating the number of sequence reads corresponding to each species. In human adults and adult cows, the intestinal microbiome has been studied by metagenomic analysis and is reported to be composed of the phyla Firmicutes (RA: 64\%, 81.9\%), Bacteroidetes (23\%, 5.4\%), Proteobacteria (8\%, 9.6\%) and Actinobacteria (3\%, 2.8\%) [1, 5]. The intestinal microbiome in calves at 36 days of age was reported to be composed of Firmicutes (49.1\%), Bacteroidetes (42.3\%) and Proteobacteria (4.1\%) [3]. Nonetheless, there have been no reports on the development of the intestinal microbiota in newborn calves by means of the metagenomic method.

Lactobacillus spp. are often administered to calves as a probiotic supplement that supports the health and growth of veal calves $[3,6]$. Using metagenomic analysis and the selective agar plate culture method, in this study, we explored the colonization of newborn calves' intestines by Lactobacillus spp.

Five clinically healthy newborn Holstein calves were used in this study. The calves were born at the full term of the gestational period on the Rakuno Gakuen University Farm. The calves were fed mother's fresh colostrum within $12 \mathrm{hr}$ after birth, and after that, they were fed $2 l$ of a commercially available milk replacement (Meiji Feed Co., Ltd., Tokyo, Japan) twice a day during the observation period.

Samples were collected from rectal feces a day after birth (day 1) and 7 days after birth (day 7). The feces on day 1 were not meconium. The collected samples were immediately cultured on agar plates for the detection of bacteria, and the rest of the samples were stored at $-30^{\circ} \mathrm{C}$ until DNA extraction.

DNA was extracted from the fecal samples using the PowerSoil ${ }^{\circledR}$ DNA extraction kit (Mobio Technologies, Vancouver, Canada). For amplification of the $16 \mathrm{~S}$ ribosomal RNA (rRNA) gene, $1 \mu l$ of the extracted DNA served as the template in subsequent PCR with a primer set (784F: 5'-AGG ATT AGA TAC CCT GG TA-3' and 1061R: 5'-GGA TTA GAT ACC CTG GTA-3') targeting the V5-V6 region of the 16S rRNA gene. Deep sequencing of the amplicon was performed on an Ion PGMTM sequencer using a

*Correspondence to: Hagiwara, K., Veterinary Medicine Rakuno Gakuen University 582 Bunkyodai, Ebetsu-shi, Hokkaido 069-0836, Japan. e-mail: k-hagi@ rakuno.ac.jp

(C2017 The Japanese Society of Veterinary Science

This is an open-access article distributed under the terms of the Creative Commons Attribution Non-Commercial No Derivatives (by-ncnd) License <https://creativecommons.org/licenses/by-nc-nd/4.o/>. 

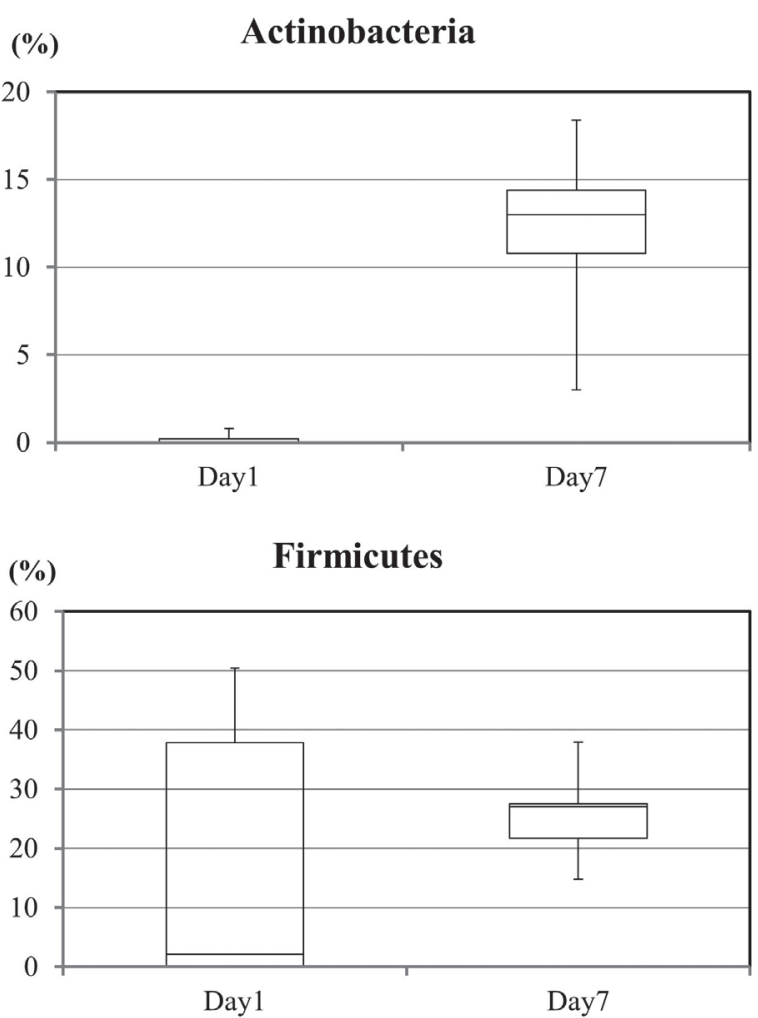

(\%)

Bacteroidetes

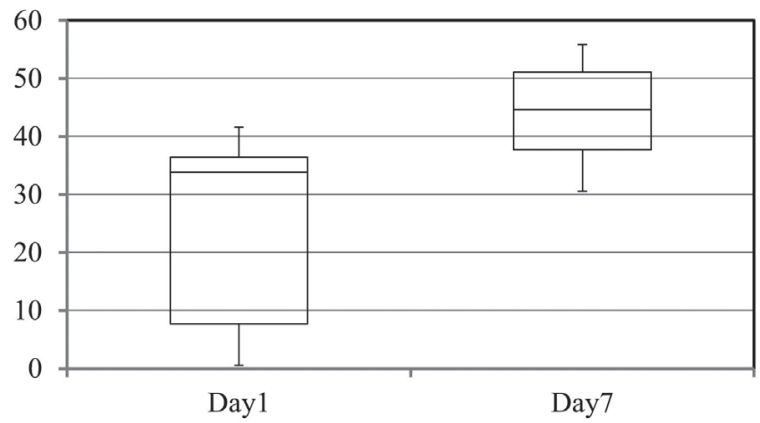

(\%)

Proteobacteria

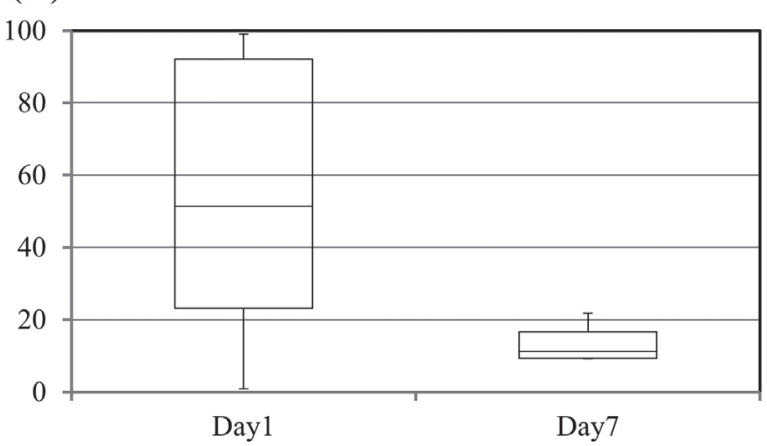

Fig. 1. The relative abundance of intestinal bacteria according to metagenomic analysis. The figure shows changes in the relative abundance of 4 phyla in feces from calves as determined by metagenomics method.

318 chip and an Ion PGM ${ }^{\mathrm{TM}}$ Sequencing 400 Kit (Thermo Fisher Scientific, Waltham, MA, U.S.A.). The resulting sequences were analyzed by means of the QIIME pipeline [1].

Fecal samples were diluted with a buffer that consisted of $0.45 \mathrm{~g} \mathrm{KH}_{2} \mathrm{PO}_{4}$ (Kishida Chemical, Osaka, Japan), $1.68 \mathrm{~g}$ $\mathrm{Na}_{2} \mathrm{HPO}_{4} \cdot 12 \mathrm{H}_{2} \mathrm{O}$ (Kanto Chemical, Tokyo, Japan), $0.05 \mathrm{~g}$ L-cysteine $\mathrm{HCl} \cdot \mathrm{H}_{2} \mathrm{O}, 0.05 \mathrm{~g}$ Tween ${ }^{\circledR} 80$ and $0.1 \mathrm{~g}$ agar in $100 \mathrm{~m} l$ of distilled water. Samples were subjected to serial 10 -fold $(\mathrm{w} / \mathrm{v})$ dilutions with the above-mentioned dilution buffer and vortexed. The Lactobacillus spp. colonies were counted after the samples were cultured on modified Lactobacillus selection (LBS) agar plates (Nissui Pharmaceutical, Tokyo, Japan). The results were expressed as the number of colony-forming units (CFUs) per g of fecal samples. Statistical analysis was performed using SPSS version 11.5 (SPSS Inc., Chicago, IL, U.S.A.). The RAs and colony counts in the microbiota between day 1 and day 7 after birth were calculated, and significance of the differences was analyzed by Student's $t$-test.

The sequencing generated a dataset consisting of filtered high-quality classifiable $16 \mathrm{~S}$ rRNA gene sequences with a mean \pm standard error (SE) of 14,317 $\pm 3,967$ sequences per sample.

The metagenomic analysis determined that several phyla were present in the microbiome. The detected bacteria could be subdivided into 4 phyla, namely Actinobacteria, Bacteroidetes, Firmicutes and Proteobacteria (Fig. 1). In samples from the first feces on day 1 , the RAs $(\%$, mean \pm SE) of these phyla were $0.2 \pm 0.14,18.1 \pm 9.7,24.0 \pm 7.4$ and 53.4 \pm 17.0 , respectively. In fecal samples obtained on day 7, the RAs of Actinobacteria, Bacteroidetes, Firmicutes and Proteobacteria were 11.9 $\pm 2.3,25.8 \pm$ $3.4,43.9 \pm 4.1$ and $13.6 \pm 2.2$, respectively. These results showed that the intestinal microbiota of newborn calves was composed of 4 phyla: Actinobacteria, Bacteroidetes, Firmicutes and Proteobacteria. The RAs of these 4 phyla changed between the 1st and 7th days after birth. All the calves were managed in the same breeding environment and given the same milk replacement diet. Nevertheless, differences in the composition of the intestinal microbiome among individual calves were observed in the day 1 feces. The individual differences in the taxonomic composition of the intestinal microbiota among the calves were smaller at 7 days after birth.

Seven families of bacteria rose to dominance in the microbiota of the tested samples during the first 7 days, as follows: families Bifidobacteriaceae and Coriobacteriaceae in phylum Actinobacteria; families Lactobacillaceae, Streptococcaceae and Veillonellaceae in phylum Firmicutes; family Bacteroidaceae in phylum Bacteroidetes; and family Enterobacteriaceae in phylum Proteobacteria (Table 1). Bifidobacteriaceae were undetectable, and the RA of Coriobacteriaceae was $0.2 \pm 0.14$ on day 1, while their RAs on day 7 were $2.3 \pm 0.29$ and $9.3 \pm 2.3$, respectively. The RAs of Lactobacillaceae, Streptococcaceae and Veillonellaceae changed from $0.14 \pm 0.13,0.16 \pm 0.07$ and $0.08 \pm 0.04$ on day 1 to $11.3 \pm 5.1,4.7 \pm 2.2$ and $7.1 \pm 1.6$ on day 7 . The RA of Bacteroidaceae increased from $4.2 \pm 2.1$ on day 1 to $22.2 \pm 4.6$ on day 7, and that of Enterobacteriaceae decreased 
Table 1. The relative abundance values of intestinal microbial taxa in newborn calves

\begin{tabular}{|c|c|c|c|c|c|}
\hline \multirow{2}{*}{ Phylum } & \multirow{2}{*}{ Family } & \multicolumn{2}{|c|}{ Day 1} & \multicolumn{2}{|c|}{ Day 7} \\
\hline & & Average (\%) & SE & Average (\%) & SE \\
\hline \multirow[t]{2}{*}{ Actinobacteria } & Bifidobacteriaceae & 0 & 0 & 2.3 & 0.29 \\
\hline & Coriobacteriaceae & 0.20 & 0.14 & 9.32 & 2.30 \\
\hline \multirow[t]{5}{*}{ Bacteroidetes } & Bacteroidaceae & 4.18 & 2.07 & 22.22 & 4.59 \\
\hline & Prevotellaceae & 3.70 & 3.31 & 1.28 & 1.12 \\
\hline & Rikenellaceae & 1.26 & 1.13 & 0 & 0 \\
\hline & Bacteroidales $524-7$ & 2.02 & 1.81 & 0 & 0 \\
\hline & Bacteroidales ratAN060301C & 5.12 & 4.56 & 1.70 & 0.54 \\
\hline \multirow[t]{7}{*}{ Firmicutes } & Lactobacillaceae & 0.14 & 0.13 & 11.26 & 5.08 \\
\hline & Streptococcaceae & 0.16 & 0.07 & 4.74 & 2.20 \\
\hline & Clostridiaceae & 4.96 & 4.00 & 0.06 & 0.02 \\
\hline & Clostridiales Family XIII Incertae Sedis & 0.24 & 0.21 & 1.54 & 0.96 \\
\hline & Lachnospiraceae & 6.44 & 2.78 & 8.4 & 2.33 \\
\hline & Ruminococcaceae & 6.82 & 3.75 & 5.62 & 2.19 \\
\hline & Veillonellaceae & 0.08 & 0.04 & 7.12 & 1.62 \\
\hline \multirow[t]{3}{*}{ Proteobacteria } & Alcaligenaceae & 0.08 & 0.07 & 2.02 & 0.59 \\
\hline & Enterobacteriaceae & 51.3 & 17.23 & 10.54 & 2.82 \\
\hline & Pasteurellaceae & 1.48 & 1.32 & 0.64 & 0.21 \\
\hline
\end{tabular}

The data show the taxonomic levels of the detected microbes (more than $1 \%$ of RA) in newborn calves on day 1 and day 7.

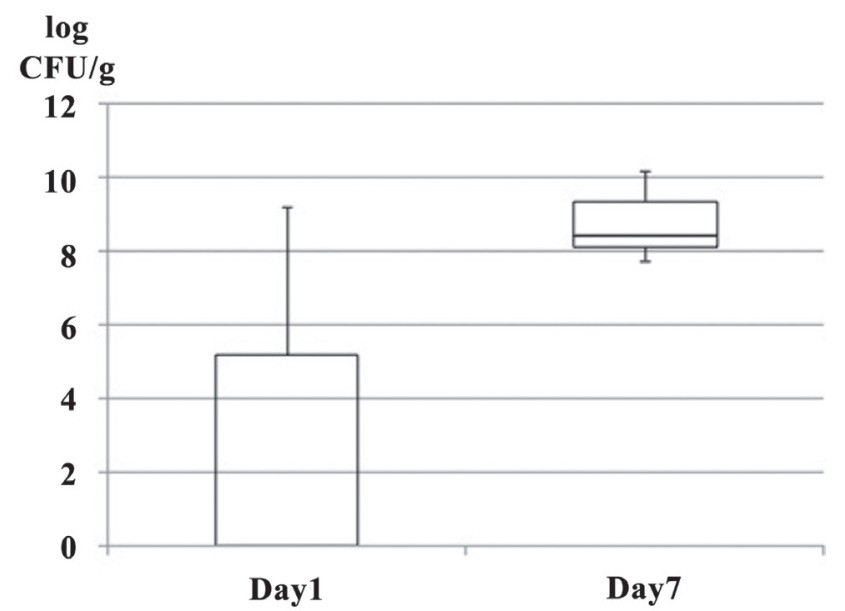

Fig. 2. Abundance of Lactobacillus spp. in feces from calves as determined by the culture method (CFU/g feces).

from $51.3 \pm 17.2$ on day 1 to $10.5 \pm 2.8$ on day 7 , respectively (Table 1$)$. The RAs of the following bacterial families increased significantly from day 1 to day 7: Bifidobacteriaceae $(P<0.001)$, Coriobacteriaceae $(P<0.01)$ and Veillonellaceae $(P<0.01)$, while that of Enterobacteriaceae decreased with borderline significance $(P=0.07)$.

One study in adult cattle reveals that Firmicutes dominate the intestinal microbiome, representing $81.9 \%$ of all sequence reads, followed by Proteobacteria (9.6\%), Bacteroidetes (5.4\%) and Actinobacteria (2.8\%) [4]. In the present study, the composition of the intestinal microbiome in the first feces varied among newborn calves born under the same environmental conditions. Therefore, some bacteria contained in the mother's colostrum may contribute to the composition of the intestinal microbiota of newborn calves.

The number of bacterial CFUs on the LBS agar plates increased in all the calves from a mean value of $2.87 \mathrm{log}$ CFU/g on day 1 to $8.76 \log \mathrm{CFU} / \mathrm{g}$ on day 7 (Fig. 2). Interindividual variability in the detected bacterial species was observed on day 1 , but the microbiomes of the calves were more homogeneous on day 7. The number of bacterial CFUs on LBS agar, which is selective for Lactobacillus spp., increased from day 1 to day 7. CFUs in the feces of calves at 7 days old reached the copy number similar to the amount reported for 12-day-old calves [6]. This result is similar to that reported for human infants [4]. The above change corresponds to the results of the metagenomic analysis. In the present study, we detected Lactobacillus spp. in the first feces samples from only 2 of 5 calves, but the population increased to more than $10^{7} \mathrm{CFU} / \mathrm{g}$ on day 7 . Therefore, the intestinal colonization of newborn calves by Lactobacillus spp. took place during the first 7 days of life.

The intestinal microbiome of newborn calves was found to comprise genetic material from the phyla Actinobacteria, 
Bacteroidetes, Firmicutes and Proteobacteria according to metagenomic analysis. The intestinal microbiome was characterized by an increase in the RA of Firmicutes (including families, such as Lactobacillaceae) during the first week after birth. Thus, the first week of life may be an important period for the intestinal colonization of calves by Lactobacillus spp.

ACKNOWLEDGMENTS. This study was supported in part by a Grant-in-Aid for Scientific Research from JSPS KAKENHI (Grant Number 23580427). We thank Mr. Taku Okamura and the staff of Rakuno Gakuen University Farm for their expert technical assistance.

\section{REFERENCES}

1. Frank, D. N., St Amand, A. L., Feldman, R. A., Boedeker, E. C., Harpaz, N. and Pace, N. R. 2007. Molecular-phylogenetic characterization of microbial community imbalances in human inflammatory bowel diseases. Proc. Natl. Acad. Sci. U.S.A. 104: 13780-13785. [Medline] [CrossRef]

2. Lukás, F., Koppová, I., Kudrna, V. and Kopecný, J. 2007. Postnatal development of bacterial population in the gastrointestinal tract of calves. Folia Microbiol. (Praha) 52: 99-104. [Medline] [CrossRef]

3. Meale, S. J., Li, S., Azevedo, P., Derakhshani, H., Plaizier, J. C., Khafipour, E. and Steele, M. A. 2016. Development of Ruminal and Fecal Microbiomes Are Affected by Weaning But Not Weaning Strategy in Dairy Calves. Front. Microbiol. 7: 582. [Medline] [CrossRef]

4. Mitsuoka, T. 2014. Establishment of intestinal bacteriology. Biosci. Microbiota Food Health 33: 99-116. [Medline] [CrossRef]

5. Rudi, K., Moen, B., Sekelja, M., Frisli, T. and Lee, M. R. 2012. An eight-year investigation of bovine livestock fecal microbiota. Vet. Microbiol. 160: 369-377. [Medline] [CrossRef]

6. Timmerman, H. M., Mulder, L., Everts, H., van Espen, D. C., van der Wal, E., Klaassen, G., Rouwers, S. M., Hartemink, R., Rombouts, F. M. and Beynen, A. C. 2005. Health and growth of veal calves fed milk replacers with or without probiotics. J. Dairy Sci. 88: 2154-2165. [Medline] [CrossRef]

7. Vlková, E., Trojanová, I. and Rada, V. 2006. Distribution of bifidobacteria in the gastrointestinal tract of calves. Folia Microbiol. (Praha) 51: 325-328. [Medline] [CrossRef] 